\title{
A Bibliometric Analysis of the HICSS Software Technology Track
}

\author{
Tor-Morten Grønli \\ Kristiania University College, \\ Dep. Technology, Oslo, Norway \\ tor-morten.gronli@kristiania.no
}

\author{
Tim A. Majchrzak \\ University of Agder, \\ Kristiansand, Norway \\ timam@uia.no
}

\author{
Hermann Kaindl \\ TU Wien, Inst. of Computer Tech., \\ Vienna, Austria \\ hermann.kaindl@tuwien.ac.at
}

\begin{abstract}
The HICSS Software Technology track has a long tradition and many papers have been published as part of its history. Its impact in terms of citations, paper contributions, author share and community impact does not yet seem to have been investigated, though. In particular, software technology has evolved, and as it stands, it is of primary importance for mobile computing, the Internet-of-Things and Cyber-physical Systems. Hence, the development of these topics and the related impact of this track are of particular interest. In this paper, we present a bibliographic analysis as a first step towards such an investigation. We found that the history of the track is indeed noteworthy. Our results include finding a few extremely much cited papers, some curious tendencies, and a generally favourable outlook for HICSS. We found that indeed many papers in the history of this HICSS track addressed mobile technology and are highly cited. Together with providing insight into track, paper and author impact, we also raise questions worth of further investigation to understand the role of the HICSS Software Technology track and its impact on the academic field and society.
\end{abstract}

\section{Introduction}

HICSS is a major conference series, and for more than 20 years its track of Software Technology (ST) has been an important contributor to its success [1]. The track has evolved in line with technological trends and covered them broadly. Moreover, the ST track has served as a window to the technical disciplines, attracting, for example also computer scientists and help bridging the academic field between information systems and computer science at HICSS.

Recently, we have seen rising importance of software engineering for mobile computing, the Internet-of-Things (IoT) and Cyber-physical Systems (CPS) [2]. That is why we have introduced a new minitrack to the Software Technology track, which in its 2020 edition includes all of these topics. It builds on the Mobile App Development minitrack introduced in 2016 [3], continued in 2017 [4], and extended to Software Development for Mobile Devices, Wearables, and the Internet-of-Things for 2018 [5] and 2019 [6].

Information technology and software engineering as research fields have seen dramatic changes over the last decades and the rapid evolvement continues. In addition, it is a clear tendency for all major research fields to make use of technology or automation for creating new possibilities. There is a need for a comprehensive understanding of these phenomena and we are curious, how "our" topics will evolve and, whether our minitrack covering them will have impact on their evolvement and what future directions to take. Hence, we would like to better understand the historical evolvement of such trends and the impact of the Software Technology track over the years. We are also curious to learn about the history of the track as a whole. Getting a comprehensive picture is the precondition for scrutinizing selected topics. We expect to shed light on the development of the software technologies field in a holistic way. The bibliometric analysis in this paper is a first step towards an investigation for getting an improved understanding.

Bibliometric analyses have been made for a long time. However, the tools for carrying them out have improved significantly through the availability of Web-based services, such as the Web of Science, Scopus, and Google Scholar [7]. While it can be discussed what should actually be measured, and who would benefit from such analyses [8], the possibility of quantifying impact is attractive to many. At least, these analyses can satisfy curiosity, but in many cases also serve more serious endeavours, such as making promotion decisions, and judging research performance in general [9]. Moreover, bibliometric analyses are particularly suited to explore the history of a topic of scientific interest [10].

With our study, we seek to answer the following research question: 
What is the HICSS Software Technology impact, and to what extent do the minitrack of Mobile, Internet-of-Things and Cyber-physical Systems mirror the overarching themes in the track?

In Section 3, we expand it to several questions to structure our study. We seek to contribute to the understanding of the history of HICSS, of the field of software technology within computer science and information systems research, and to the placement of "our" topics within these fields.

The remainder of this paper is structured as follows. Section 2 provides a historic overview of the Software Technology track. Based on the research design as described in Section 3, the bibliometric analysis follows in Section 4. After that, a detailed discussion of the results is given in Section 5, before we draw a conclusion in Section 6.

\section{A Brief Account of the History}

The HICSS conference series was initiated long time ago, in 1968. In 2014, its history was reviewed [1]. After 50 years, the jubilee conference HICSS-50 had over 1000 participants and over 500 paper presentations [11].

We are not the first to look back at the history of this conference series. Thomas [12], for example, presented on "Reflections on Twenty Years of Electric Power Research at HICSS", but there was no bibliometric analysis. Nonetheless, the general idea - specifically considering a topical part of HICSS - is comparable to ours. Marjanovic and Dinter [13] presented a text-mining-based analysis of a minitrack (Business Intelligence) having been running for over 25 years. Dittes et al. [14] gave an overview of eleven years of the Knowledge Management track; interestingly, it appeared in a journal and not in the HICSS Proceedings.

For more than 20 years, the track on Software Technology has been an important contributor to the success of the HICSS conference series. For the scope of our paper, we will look back exactly 20 years, starting with the 2000 edition of the track. Despite the Internet having being used by scientists much earlier, it becomes harder and harder to retrace scientific conferences beyond the transition to the new millennium. Conference Web sites back then looked much differently and the scientific output (in quantitative terms) was much lower, with typical scholars ${ }^{1}$ publishing much fewer papers per year than they do now. ${ }^{2}$ Even though there is good indexation,

\footnotetext{
${ }^{1}$ i.e. not scholars like Paul Erdôs.

${ }^{2}$ Likely, the number of scholars particularly from regions rather recently focussing on science has increased, and new tools enable more rapid paper writing; however, substantiating such claims would
}

a lack of original sources make it hard to verify the results in databases. For example, the computer science bibliography DBLP lists HICSS starting from 1994 [15]. However, occasionally tracks are merely numbered instead of listed by name.

HICSS is also known for consistency and tradition; this also applies to the ST track. The current track chairs, Rick Kazman of the University of Hawaii at Manoa, and Gul Agha of the University of Illinois, Urbana serve since 2007 and 2005, respectively. Topically, the ST track has seen a number of minitracks with fundamental topics, which sustained (or still sustain) for extended periods. Other minitracks follow current topics and are discontinued or adapted after a few years.

Within the ST track, we have organized a series of related minitracks starting in 2016 [3, 4, 5, 6]. The idea behind initiating this series of minitracks was twofold: First, we wanted to contribute to keep HICSS interesting for computer scientists and for those researchers within information systems who conduct research "between the realms". Second, we sought to give mobile computing and related topics a home. Mobile computing and predecessors of research on IoT have been featured at HICSS for decades, but giving them an explicit focus appeared to be reasonable to us, following the current developments in the field, e.g., regarding app-enablement [16]. Our minitrack series combines tradition and timeliness. While we run it now in its fifth year, with hopefully many more to come, we have adapted it two times already to include current developments and to stay relevant to the community.

\section{Research Design}

The research design section in this paper consists of two major parts. First, we give an overview of the research questions resulting from a breakdown of our overarching research question. Second, we present our method to explain our approach, justify it, and to make it repeatable for future studies.

\subsection{Research Questions}

To pursue the overarching research question presented in the introduction, we have chosen to highlight a number of subordinate research questions to be able to address the individual issues identified:

- RQ1: How did the Software Technology track develop over time considering the number of minitracks, number of papers, and citations?

be complex enough for a paper of its own. We will, thus, not even try to do this here. 
- RQ2: Does the average number of citations per paper over the years follow the usual pattern of older papers having been able to collect more citations over time?

- RQ4: Are papers from authors who have published in the same minitrack over more than one year cited more often?

- RQ5: Are the average numbers of citations dealing with mobile, IoT or CPS higher than the average numbers of citations of all papers?

- RQ6: Is the impact of HICSS in terms of citations per paper comparable to dedicated software engineering or related conferences?

- RQ7: How did the impact of papers dealing with mobile, IoT or CPS perform over time?

- RQ8: What is the impact of our minitrack in the scope of HICSS ST?

- RQ9: Can an outlook be given based on the bibliometrics of the ST track?

\subsection{Method}

In the following, we explain our method in terms of procedures to answer the nine research questions. HICSS has maintained a relatively systematic archive of conference programs. The programs from all the HICSS conferences from 2000 to the most recent one in 2019 was extracted from the DBLP Web site, cleaned up and put into a systematic format. Minitracks with title, their papers, author numbers per paper, sum of citations, number of papers on the minitrack, average citation number and length of title in words were extracted semi-manually for each year since 2000 and onward. The numbers of citations per paper were collected through manual Google Scholar look-ups for each individual paper. The whole process is designed along the lines of previous work [17].

A script was created to perform simple bibliometric analyses. Regular expressions helped to accelerate manual tasks. Microsoft Excel was used as the primary tool for analysis together with its Visual Basic script editor. Number of minitracks per year, citations per year, papers per year, average number of citations per minitrack per year, number of authors per paper per year, average title length per paper and average number of citations per paper per year were all collected through writing Visual Basic code in the Excel Macro editor and applying it to the data sheets.

Unfortunately, no download information is publicly available for HICSS papers. $^{3}$ Counts of unique

\footnotetext{
${ }^{3}$ For the last few conferences, which are included in the AIS eLibrary [18] such figures are available. However, we have no information how reliable these are. Possibly, these download numbers
}

downloads can be used as a rough measure of interest in a paper, as a paper that has been downloaded more often is likely to have been read by more people than a paper with a low download count. This information could have been even more useful to analyse which HICSS papers have sparked the most interest.

Citation analyses were conducted using Google Scholar. The citation counts for each HICSS publication had to be retrieved manually as Google Scholar does not allow for automatic retrieval of information using Web robots. The manual process revealed that the quality of the paper meta-information of the indexed HICSS papers are somewhat irregular with mistakes, for example with family names treated as surnames, variations in the use of the conference name, etc. However, the manual process offered the chance to clean up such irregularities directly.

Moreover, we could also scrutinize sources of errors. In some cases, papers had been republished with a very similar (or even the same) title. While this is a questionable practice, it would have lead to wrong citation counts if no corrections had been applied. We also recognize that data for the contributions from 2017, 2018 and 2019 are not included due to their very recent publication date making the impact of them citation wise very low so far, thus we would not be able to give a good impression of these papers.

\section{Bibliometric Analysis}

The results of the bibliometric analysis are presented in the following subsections.

\subsection{Minitracks and Papers}

Looking back at the years starting from 2000, the number of minitracks was on average 10 tracks per year. As illustrated in Figure 1, the years with the most minitracks include 2003 and 2005, with 15 and 14 minitracks, respectively. Then, for the next decade there was not much variation in this respect until 2019, when the number increased again to 15 . The mean value is shown as a dotted line.

During the same period, the numbers of papers per year has varied between a low of 34 in 2008 and the peak at 102 in 2003. The details are given in Figure 2.

We consider a stable number of papers each year as a quality sign of continuous broad support of the track and gathering of the community. During the last decade, the paper numbers have been very stable. With the general HICSS policy of keeping the acceptance rate well below 50 percent, this contributes to keep the quality at a high

can be analyzed in a few years. 


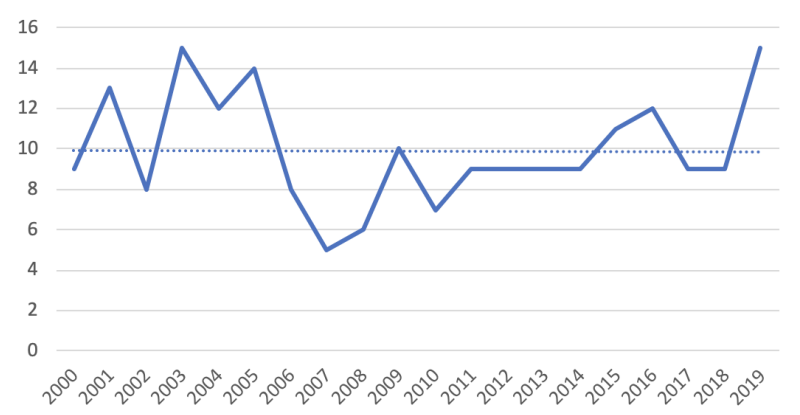

Figure 1. Number of Minitracks per Year

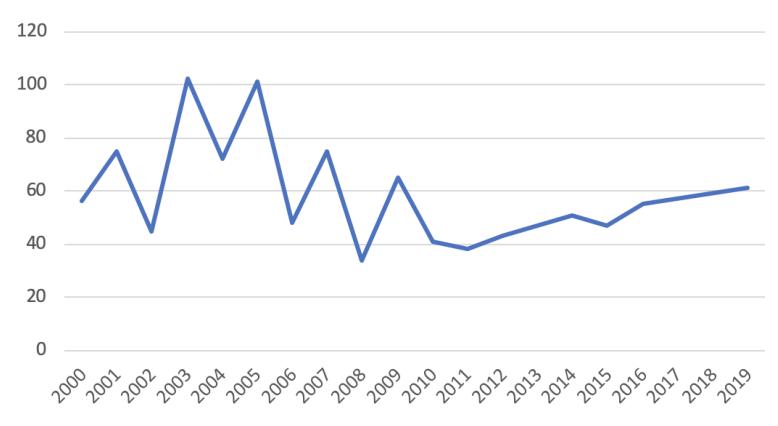

Figure 2. Papers per Year

level.

\subsection{Publication Title Length}

An interesting observation is the low variation in paper length during the years since 2000, averaging at 10, as shown in Figure 3, and with a standard deviation of 2. This indicates that authors favour a medium to short length of titles. Interestingly, short paper titles appear to have an advantage over long ones [19]. From observing the titles in the data material, it is best characterized as descriptive and informative. Furthermore, this observation highlights that there is neither an increasing nor a decreasing trend towards more or less verbose paper titles.

Figure 4 shows the median for the same data set. It almost resembles the averages, indicating no noticeable effect of outliers.

\subsection{Number of Authors per Paper}

The number of authors per paper is on average 2.62 over all the years taken into account here. Two dips, in 2003 and 2005, are the only deviations from this average. However, the trendline indicates that there is an increasing tendency, and the average for the last 10 years is 2.96. Figure 5 gives the full details. Figure 6 is based on the same data but shows the median.

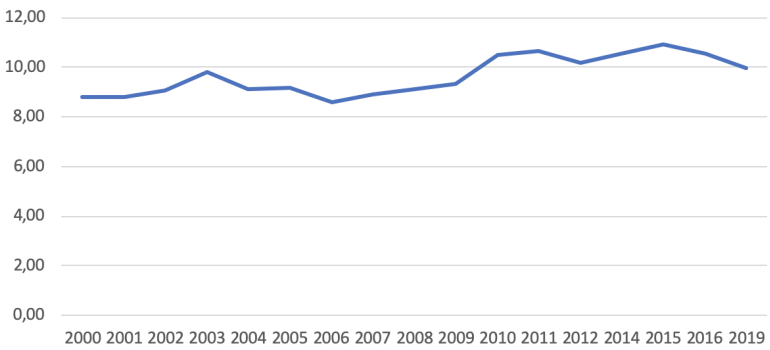

Figure 3. Average Title Length per Paper per Year

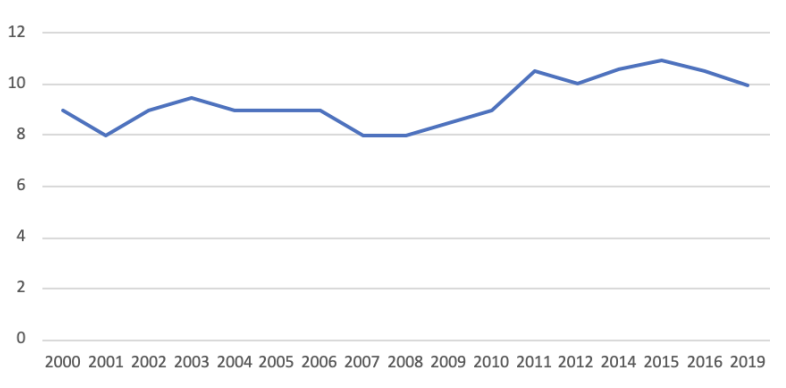

Figure 4. Median Title Length per Paper per Year

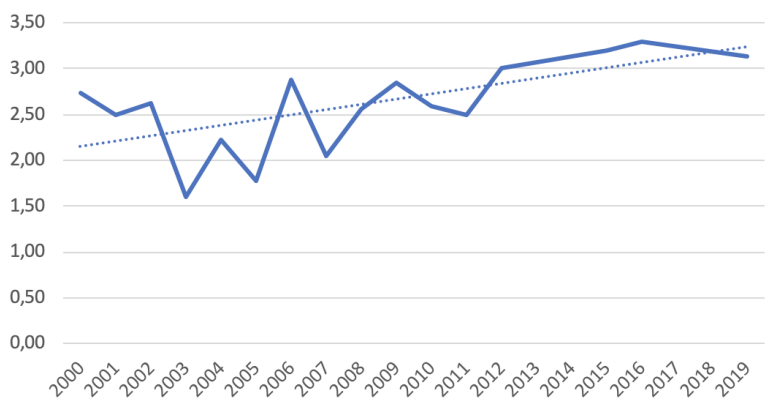

Figure 5. Average Number of Authors per Paper per Year

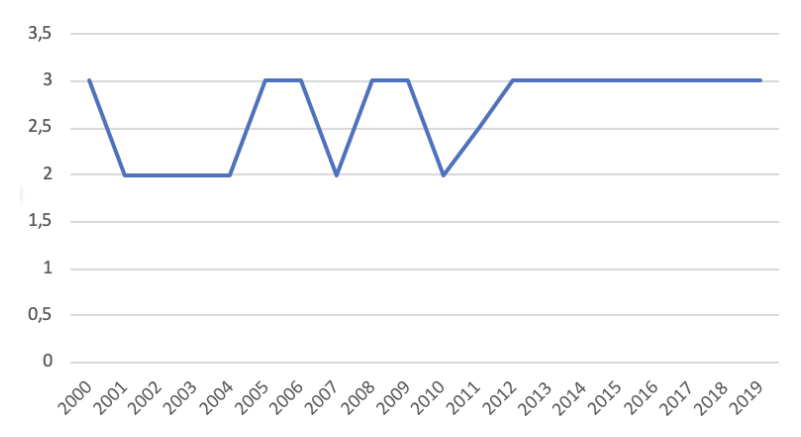

Figure 6. Median Number of Authors per Paper per Year

\subsection{Number of Citations}

The number of citations indicates significance and interest and, hence, impact. As such, the number of 
citations is considered an important indicator of success. Isolating the numbers, and only looking at the ST track, some interesting observations become visible in Figure 7.

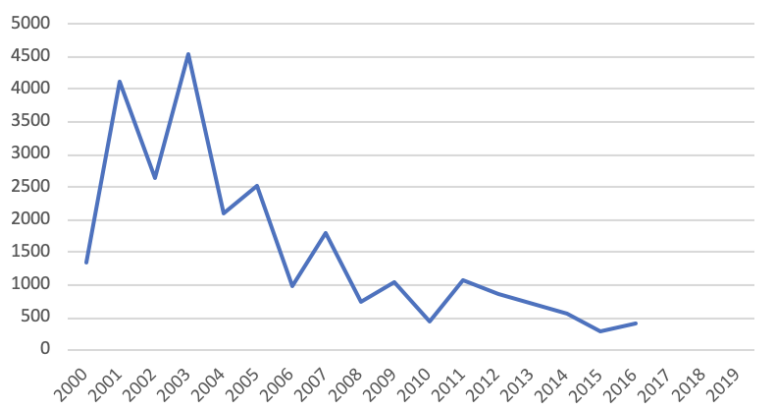

Figure 7. Citations in Total per Year

What can be observed is the expected trend of older papers having generated higher citation numbers, on average, as compared to more recent publications. This is an obvious and expected observation, as it takes time to acquire citations, and older work has a longer exposure time.

The citation data of the year 2000 has been cleaned from one outlier in the dataset. It has achieved a stunning 17,000 citations (which is discussed below). The average number of citations per year is over this period 2,669, and for the last 10 years it is 1,606 . As mentioned already above, all citation numbers are manually acquired from Google Scholar, and we disregard the ongoing academic discussion of reliability vs. manipulability of citation scores [20].

When looking at the number of citations per year based on the median (Figure 8), we can observe that a paper typically has around 10 citations, with papers from more recent years having slightly fewer citations, as expected due to the shorter time period since publication. From the earlier years, 2004 represents a year with high number of citations on many papers, bringing the median to 25 .

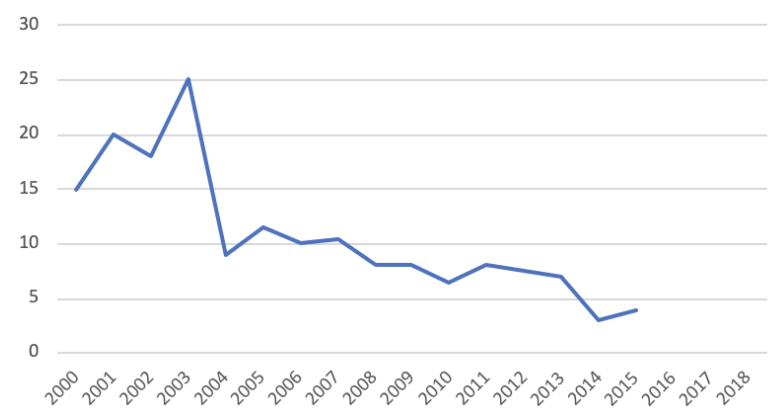

Figure 8. Median of Citations per Paper per Year
Another perspective is to have a look at the average number of citations per minitrack. Figure 9 gives the details, with an average for the time period of 24 .

Figure 10 shows the median for the sum of citations per minitrack per year and provides a slightly different perspective one the same data as the previous figure. The trend is fairly consistent with the exception of a high number of citations on the smaller number of minitracks in 2011 .

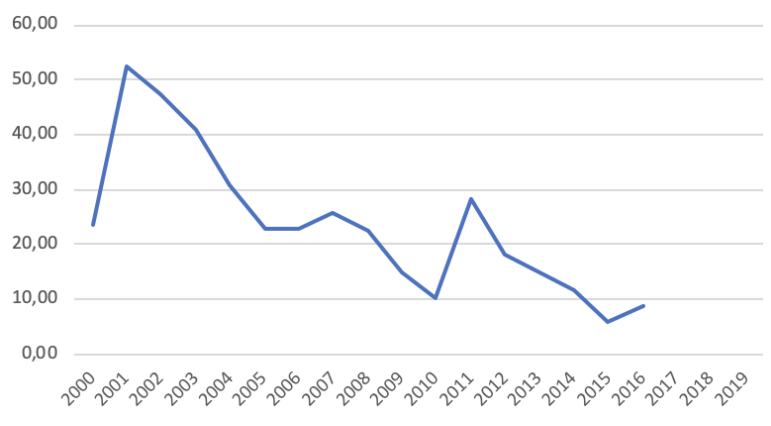

Figure 9. Average Citations per Minitrack per Year

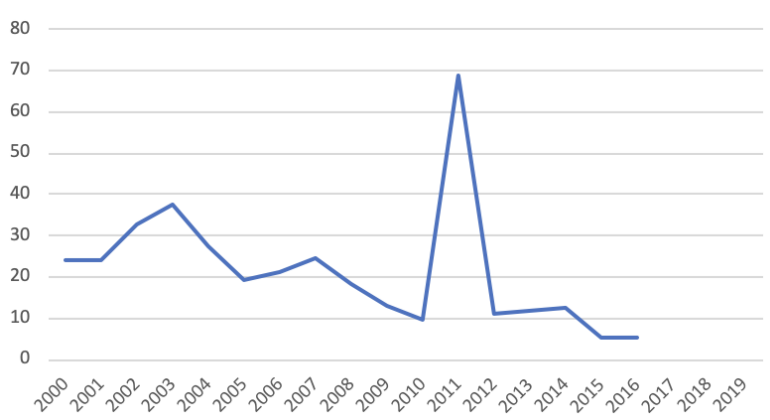

Figure 10. Median of Sum of Citations per Minitrack per Year

There are large variations between different minitracks, and there are large variations of numbers of papers per minitrack. In order to interpret the data differently, we show in Figure 11 the average numbers of citations per paper per year, independently of the minitrack they have been presented in.

The average number of citations per paper per year is 25 over the time period from 2001 to 2017. While 2011 was a year with a low average citation number of 10 , there was an increase in 2012 (to 28). Hence, there was a slight fluctuation.

\subsection{Conference Comparison}

The data for our conference comparison in Table 1 were pulled from the international SCImago Journal and Country Rank [21], based on developed criteria and 


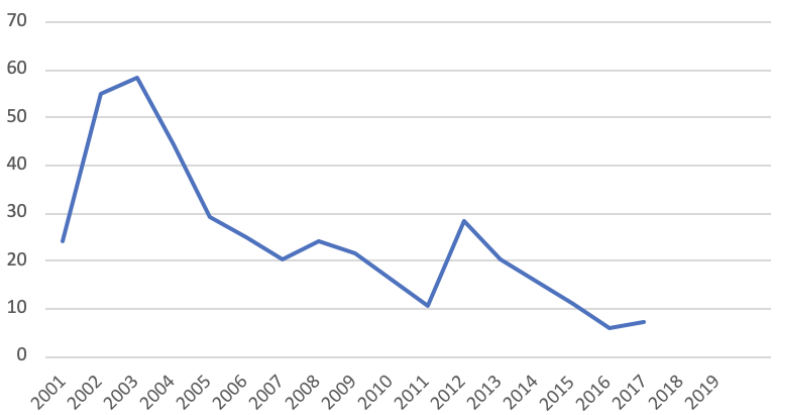

Figure 11. Average Number of Citations per Paper per Year

indexing in Scopus. The number of citations received by the papers is divided by the total number of papers published. The numbers for measuring citations per paper were the reported numbers for the last three years originating from 2018, i.e., covering the period of 2016-2018. Different time periods and lengths will possibly influence this number, but it highlights recent relevant scores. By comparing to premier dedicated conferences, we can see the relative position and impact.

Table 1. Conference Comparison

\begin{tabular}{lcc}
\hline Conference & $\begin{array}{c}\text { Citations } \\
\text { per paper }\end{array}$ & h-index \\
\hline $\begin{array}{l}\text { International Conference on Software } \\
\text { Engineering (ICSE) }\end{array}$ & 4.00 & 125 \\
\hline $\begin{array}{l}\text { Conference on Human Factors in } \\
\text { Computing Systems (CHI) }\end{array}$ & 3.74 & 165 \\
\hline $\begin{array}{l}\text { International Joint Conference on } \\
\text { Artificial Intelligence (IJCAI) }\end{array}$ & 2.54 & 109 \\
\hline $\begin{array}{l}\text { Hawaii International Conference on } \\
\text { System Sciences (HICSS) }\end{array}$ & $\mathbf{1 . 2 7}$ & $\mathbf{7 2}$ \\
\hline $\begin{array}{l}\text { Electronic Components and Technology } \\
\text { Conference (ECTC) }\end{array}$ & 1.21 & 65 \\
\hline $\begin{array}{l}\text { Proceedings of the Annual International } \\
\text { Conference on Mobile Computing and } \\
\text { Networking, MOBICOM }\end{array}$ & 1.03 & 49 \\
\hline
\end{tabular}

We use the h-index to measure impact for minitrack and paper. The h-index is an index to quantify an individuals scientific research output based on the set of the researcher's most cited papers and the number of citations that they have received in other people's publications [22]. The h-index numbers for the conferences listed in the table are as reported from the ranking page of SC Imago [21].

\subsection{Top-cited Papers}

Apart from average numbers, it is interesting to have a look at the top-cited papers from the HICSS Software
Technology track. Table 2 provides the top ten list. Particularly the more than 17,000 citations of the top paper are impressive. The second most-cited paper has some 1,700 citations, but this figure is already an order of magnitude smaller. The citation numbers of the other papers on this top ten list are on average 300-500, i.e., still in the hundreds. All these top-cited papers are from the years 2000 to 2003, with only two exceptions. Again, this is not unexpected as it takes time to gain attention and impact. Nonetheless, this might have been a particularly successful time for the track, as it does not seem likely for papers from the successor years to catch up if they are not already close with their citation count.

It is even more interesting to have a look at the topics addressed by these papers. Nearly all of them relate to mobility! The top-cited paper appeared in a minitrack called Mobile Computing and Wireless Networks, i.e., a minitrack that about 20 years ago must have dealt with similar topics as our minitrack today.

\subsection{Mobile Technology Minitrack}

The Mobile Technology minitrack combines tradition and timeliness. While in 2020 it is run in its fifth year, we have adapted it two times already to include current developments and stay relevant to the community. Our adaptation of the minitrack to first include Internet-of-Things (IoT) and then, this year, to include Cyper-physical Systems (CPS) is in a way mirroring the diversity and changing world of software engineering solutions. While at first mobility was seen as a separate topic and rarely included as a natural part of a software development project, it has now become main stream and the users of any application expect to be able to use it across their heterogeneous composition of devices and platforms (following concepts in user interface design such as responsiveness [23]).

Internet-of-Things then transpired to become a research stream and as technology spreads to household, manufacturing, health service, transportation to mention a few, it becomes increasingly main stream. The link to Cyber-physical Systems is short as it bridges the gap from components and devices being enriched with sensor functionality and systems being controlled by software.

Table 3 lists all papers presented as part of our minitrack so far. Quite obviously, most of them have the term mobile (or a related term such as app already) in the title.

\section{Discussion of the Results}

In the following, we discuss the results from our analysis. First, we summarise our main findings and 
Table 2. The Most Cited Papers from the HICSS Software Technology Track

\begin{tabular}{|c|c|c|c|c|}
\hline Minitrack & Paper & Authors & Year & Citations \\
\hline Mobile Computing and Wireless Networks & $\begin{array}{l}\text { Energy-Efficient Communication Protocol for } \\
\text { Wireless Microsensor Networks. }\end{array}$ & 3 & 2000 & 17,262 \\
\hline Mobile Ad-hoc Networking & GPS-Free Positioning in Mobile ad-hoc Networks & 3 & 2001 & 1,701 \\
\hline Mobile Computing and Wireless Network (STMCW) & $\begin{array}{l}\text { Multipoint Relaying for Flooding Broadcast } \\
\text { Messages in Mobile Wireless Networks }\end{array}$ & 3 & 2002 & 887 \\
\hline $\begin{array}{l}\text { Energy Management in Mobile and Pervasive } \\
\text { Computing Systems }\end{array}$ & $\begin{array}{l}\text { Exploiting Sink Mobility for Maximizing Sensor } \\
\text { Networks Lifetime }\end{array}$ & 4 & 2005 & 555 \\
\hline $\begin{array}{l}\text { Quality of Service in Mobile and Wireless Network } \\
\text { (STQSM) }\end{array}$ & $\begin{array}{l}\text { On Reducing Broadcast Redundancy in Ad Hoc } \\
\text { Wireless Networks }\end{array}$ & 2 & 2003 & 519 \\
\hline Mobile Distributed Information Systems (STMDI) & $\begin{array}{l}\text { Context-Awareness on Mobile Devices - the } \\
\text { Hydrogen Approach }\end{array}$ & 6 & 2003 & 507 \\
\hline Mobile Computing and Wireless Network (STMCW) & $\begin{array}{l}\text { New Distributed Algorithm for Connected } \\
\text { Dominating Set in Wireless Ad Hoc Networks }\end{array}$ & 3 & 2002 & 379 \\
\hline $\begin{array}{l}\text { Adaptive and Evolvable Software Systems: } \\
\text { Techniques, Tools, and Applications }\end{array}$ & $\begin{array}{l}\text { Distributed Scrum: Agile Project Management with } \\
\text { Outsourced Development Teams }\end{array}$ & 4 & 2007 & 351 \\
\hline Mobile Commerce: A New Frontier for E-business & $\begin{array}{l}\text { Wireless Commerce: Marketing Issues and } \\
\text { Possibilities }\end{array}$ & 3 & 2001 & 308 \\
\hline Mobile Computing and Wireless Network (STMCW) & $\begin{array}{l}\text { Sparse Power Efficient Topology for Wireless } \\
\text { Networks }\end{array}$ & 4 & 2002 & 206 \\
\hline
\end{tabular}

attempt to answer our research questions. Then, we lead over to open questions and research directions, including some curiosities that our work revealed. Eventually, we discuss limitations.

\subsection{Main Findings}

Revisiting the research questions we discuss them in the light of our findings. The first question we asked was: RQ1: How did the Software Technology track develop over time considering the number of minitracks, number of papers, and citations? The data in the related figures above provide related answers, but there is no particularly surprising insight.

The second questions was: RQ2: Does the average number of citations per paper over the years follow the usual pattern of older papers having been able to collect more citations over time? In spite of the small fluctuations in Figure 11, we tend to answer our second research question affirmatively, i.e., the average number of citations per paper over the years follows the usual pattern of older papers having been able to collect more citations over time.

Our third research question was on the topics of those papers in this track with the highest numbers of citations, specifically: RQ3: What are the papers with the highest number of citations and which topics do they cover? The answer is surprisingly plain. The more or less ubiquitous topic of these papers is mobility (please refer to Table 2). Despite our idea for this paper, and our minitrack as the ultimate reason for writing it, this finding, or rather this unrivalled focus on mobility, is (positively) unexpected.

Question four was: RQ4: Are papers from authors cited more often who have published in the same minitrack over more than one year? Unfortunately, analysing the data in this respect turned out to be more difficult than expected. Hence, we do not yet have an answer to RQ4, but still consider it an interesting open question for future work.

Our fifth question was focussed on the topics of our own minitrack: RQ5: Are the average numbers of citations dealing with mobile, IoT or CPS higher than the average numbers of citations of all papers? In fact, papers dealing with mobility had on average much higher numbers of citations. For the other topics listed in RQ5, we could not yet find a clear answer. This likely is owed to their recency.

Being a long running and successful conference on its own is one thing, but it is a completely different matter how it is perceived by the academic field and which impact it has. To better understand this we asked in the sixth question: RQ6: Is the impact of HICSS in terms of citations per paper comparable to dedicated software engineering or related conferences? For finding out whether the impact of the HICSS Software Technology track conference series in terms of citations per paper and h-index is comparable to dedicated software engineering conferences like ICSE and other premier conferences like CHI and IJCAI, let us have a look into Table 1 again. While the absolute premier conference series in their respective 
Table 3. All Papers Presented as Part of the Mobile Technology Minitrack

\begin{tabular}{|c|c|c|c|}
\hline Year & Title & Authors & Cit. \\
\hline 2016 & An Investigation of Usability of Push Notifications on Mobile Devices for Novice and Expert Users & 2 & 15 \\
\hline 2016 & Developing Apps for Visually Impaired People: Lessons Learned from Practice & 4 & 10 \\
\hline 2016 & $\begin{array}{l}\text { MAsCOT: Self-Adaptive Opportunistic Offloading for Cloud-Enabled Smart Mobile Applications with Probabilistic } \\
\text { Graphical Models at Runtime }\end{array}$ & 4 & 15 \\
\hline 2016 & $\begin{array}{l}\text { Meeting Quality Standards for Mobile Application Development in Businesses: A Framework for Cross-Platform } \\
\text { Testing }\end{array}$ & 2 & 14 \\
\hline 2016 & Mobile Application Developers' Platform Choice Model & 4 & 6 \\
\hline 2016 & Towards a Reference Architecture for Model-Driven Business Apps & 3 & 8 \\
\hline 2017 & Towards a Mobile Learning Environment using Reference Architectures & 4 & 4 \\
\hline 2017 & $\begin{array}{l}\text { Enriching Augmented Reality with Text Data Mining: An Automated Content Management System to Develop } \\
\text { Hybrid Media Applications }\end{array}$ & 3 & 2 \\
\hline 2017 & Exploitation and Detection of a Malicious Mobile Application & 3 & 4 \\
\hline 2017 & Comprehensive Analysis of Innovative Cross-Platform App Development Frameworks & 3 & 21 \\
\hline 2018 & Metamorphic Testing of Navigation Software: A Pilot Study with Google Maps & 3 & 10 \\
\hline 2018 & A Language-based Approach for Interoperability of IoT Platforms & 4 & 6 \\
\hline 2018 & Energy-Aware Scheduling of Conditional Task Graphs on NoC-Based MPSoCs & 3 & 3 \\
\hline 2018 & Bridging the Gap: Investigating Device-Feature Exposure in Cross-Platform Development & 2 & 7 \\
\hline 2018 & Evaluating a Graphical Model-Driven Approach to Codeless Business App Development & 1 & 6 \\
\hline 2018 & Progressive Web Apps: the Definite Approach to Cross-Platform Development? & 3 & 18 \\
\hline 2019 & The Influence of Design Updates on Users: the Case of Snapchat & 3 & 11 \\
\hline 2019 & Examining User-Developer Feedback Loops in the iOS App Store & 3 & 9 \\
\hline 2019 & Offloading for Mobile Device Performance Improvement & 3 & 6 \\
\hline 2019 & A Model-Driven Cross-Platform App Development Process for Heterogeneous Device Classes & 2 & 10 \\
\hline 2019 & Automated Testing of Motion-based Events in Mobile Applications & 2 & 8 \\
\hline 2019 & $\begin{array}{l}\text { Software Development for Mobile Computing the Internet of Things and Wearable Devices: Inspecting the Past to } \\
\text { Understand the Future }\end{array}$ & 3 & 5 \\
\hline 2019 & $\begin{array}{l}\text { Successful Mobile Application Development: Towards a Taxonomy of Domain-Specific Process Models and } \\
\text { Methodologies }\end{array}$ & 3 & 13 \\
\hline
\end{tabular}

fields ICSE, CHI and IJCAI appear to have more impact than HICSS, the important ECTC appears to have less impact. It does not seem to be possible to draw a clear picture, and specialised conference may have a higher impact. Moreover, some fluctuations can be observed with the number of minitracks over the years. The fluctuating in citation count in the first ten or so years covered is much higher, it becomes more stable with an upward trend in recent years. Thus, the comparison of conferences in individual years might look much different than it does overall. Possibly, it would also be needed to distinguish between those conferences that typically serve as starting ramps for journal extensions (HICSS for example has many post-conference fast-track opportunities) and those that publish finalized research with typically little direct follow-up work.
While it is interesting and provides insight to look back in time as to how and what effects where achieved, it is also important to keep an eye on the road ahead. Therefore, we asked in question seven: RQ7: How did the impact of papers dealing with mobile, IoT or CPS perform over time? Our data is too limited and the public HICSS bibliometric history is quite poor. This makes it yet impossible to answer this question. However as the continuation of IT solutions converge and the majority of software engineering projects start to have mobile, wearable and IoT as an integral part of any solution, this will increasingly become important to keep an eye on.

The same applies to research question number eight: RQ8: What is the impact of our minitrack in the scope of HICSS ST?. There are interesting indications of the impact in relation to the increased focus on mobility and 
Internet-of-Things in research and software projects, but our data is too limited at the moment to enable providing a concrete answer.

Finally, we asked: $R Q 9$ : Can an outlook be given based on the bibliometrics of the ST track? Against our expectation, this question is very hard to answer. There is heterogeneity in the findings, and the true impact of a publication year only becomes visible many years later. The figures indicate that there is no reason to believe in a grim future of the ST track; at the same time, a continuously high impact will require sustainability. Although impossible to prove quantitatively, it is safe to say that the track is bound to the efforts and dedication by its minitrack co-chairs in keeping the track timely according to new trends.

\subsection{Open Questions and Research Directions}

Through this research, several research questions where asked. Some could not yet be exhaustively answered, due to lack of available data. While we still were able to present interesting insights, there is much room for future work.

A much more detailed analysis of the tendency in citation count and deviation would be interesting. This could answer whether there is a downward tendency with numbers of citations. And, if there was one, it would be interesting to see if this is a general trend. Moreover, future analysis could try to shed light on where citations come from. Are HICSS papers mainly cited by other HICSS papers, by conference papers, from journal papers? And is it rather the same community citing them, or do they attract many citations across disciplines? And are the average numbers of citations dealing with mobile, IoT or CPS higher than the average numbers of citations of all papers? This could also give valuable knowledge for how to plan and conduct future organizations of the minitrack. Admittedly, such questions are very hard to answer, but also very insightful if answered.

The conference comparison is not exhaustive. Ideally, future work could guide software engineers, especially those whose work is interdisciplinary, regarding their publication activities. Additionally, it could also help HICSS to become more attractive to more technically oriented researchers.

Finally, while we could ascertain a high interest of the topics covered by our minitrack, we could not answer why this is the case. Especially, we could not indicate how such interest can be sustained and used to facilitate the impact of the minitrack.

\subsection{Limitations}

Despite being carried out rigorously and following common practices in bibliometric studies, our work has some underlying limitations that ought to be mentioned. First, we conducted much manual work and relied on little automation. While this is a quality filter at the same time, it also meant that mere typos could lead to errors, despite high effort to double check numbers.

Second, we rely on data from a number of sources. Assessing the reliability of all data is impossible, and even data that is relatively reliable might have been target to manipulation. For example, citation counts reported by Google might be correct as such, yet artificially high due to an unusually high number of self-citations from publications with comparatively low impact. Moreover, there are indications that citation statistics are prone to certain kinds of error when compiled automatically [24].

Third, much of the interpretation of the data is to some degree speculative. In many cases, we could argue that we observed a certain phenomenon and we could give an educated guess as to why it could be observed. However, it is an inherent limitation of our quantitative research method that we cannot certainly say what was the underlying reason or mechanism behind a phenomenon.

\section{Conclusion}

In this paper, we present a bibliometric analysis of the Software Technology (ST) track of the Hawaii International Conference on System Sciences (HICSS). Motivated by running our own minitrack as part of this track, we took a look at the last 20 years of the history of the ST track. Our aim was to describe its impact while allowing for a peek into its future. We found that the ST track has had significant impact in the software engineering community. It has proven to be sustainable. Mobility, as also the main topic of our minitrack, has been found to have particularly high impact.

Our paper makes several contributions. First, we provide a bibliometric analysis of 20 years of the HICSS ST track. It should help to better understand trends as well as the role and importance of the ST track for the conference as a whole. Second, we derive open questions and research findings based on the analysis. Third, we specifically provide the link to our work in the minitrack focusing on mobility and related aspects.

\section{References}

[1] "Forty seven years of HICSS - a brief history of the hawaii international conference on system sciences," in 
2014 47th Hawaii International Conference on System Sciences, pp. lii-lxxv, Jan 2014.

[2] T.-M. Grønli, A. Biørn-Hansen, and T. A. Majchrzak, "Software development for mobile computing, the internet of things and wearable devices: Inspecting the past to understand the future," in Proceedings 52th Hawaii International Conference on Systems Science (HICSS-52), AIS Electronic Library (AISeL), 2019.

[3] T. A. Majchrzak and H. Heitkötter, "Introduction to the mobile app development minitrack," in Proceedings 49th Hawaii International Conference on Systems Science (HICSS-49), p. 5682, IEEE Computer Society, 2016.

[4] T. A. Majchrzak and T.-M. Grønli, "Introduction to the mobile app development minitrack," in Proceedings 50th Hawaii International Conference on Systems Science (HICSS-50), p. 6161, AIS Electronic Library (AISeL), 2017.

[5] T. A. Majchrzak and T.-M. Grønli, "Introduction to the HICSS-51 software development for mobile devices, wearables, and the internet-of-things minitrack," in Proceedings 51th Hawaii International Conference on Systems Science (HICSS-51), p. 5686, AIS Electronic Library (AISeL), 2018.

[6] T. A. Majchrzak and T.-M. Grønli, "Introduction to the HICSS-52 software development for mobile devices, wearables, and the internet-of-things minitrack," in Proceedings 51th Hawaii International Conference on Systems Science (HICSS-52), p. 7399, AIS Electronic Library (AISeL), 2019.

[7] L. I. Meho and K. Yang, "A new era in citation and bibliometric analyses: Web of science, scopus, and google scholar," arXiv preprint cs/0612132, 2006.

[8] T. Van Leeuwen, "The application of bibliometric analyses in the evaluation of social science research. who benefits from it, and why it is still feasible," Scientometrics, vol. 66, no. 1, pp. 133-154, 2006.

[9] A. F. Van Raan, "The use of bibliometric analysis in research performance assessment and monitoring of interdisciplinary scientific developments," Technology Assessment-Theory and Practice, vol. 1, no. 12, pp. 20-29, 2003.

[10] F. G. De Bakker, P. Groenewegen, and F. Den Hond, "A bibliometric analysis of 30 years of research and theory on corporate social responsibility and corporate social performance," Business \& society, vol. 44, no. 3, pp. 283-317, 2005.

[11] "Hawai'i international conference on system sciences celebrates 50 years of research and innovation." https://manoa.hawaii.edu/kaunana/

hawaii-international-conference-onsystem-sciences-celebrates-50-yearsof-research-and-innovation/, 2019.

[12] R. Thomas, "Reflections on twenty years of electric power research at HICSS," in Bui [25], pp. 1-11.

[13] O. Marjanovic and B. Dinter, "25+ years of business intelligence and analytics minitrack at HICSS: A text mining analysis," in Bui [25], pp. 1-10.

[14] S. Dittes, S. Smolnik, M. E. Jennex, and D. T. Croasdell, "Eleven years of the knowledge management track at HICSS: an overview," IJKM, vol. 12, no. 4, pp. 51-61, 2016.

[15] "DBLP: Hawaii international conference on system sciences (HICSS)." https://dblpl.unitrier.de/db/conf/hicss/index.html, 2019.
[16] C. Rieger and T. A. Majchrzak, "A Taxonomy for App-Enabled Devices: Mastering the Mobile Device Jungle," in Revised Selected Papers WEBIST 2017 (T. A. Majchrzak, P. Traverso, K. Krempels, and V. Monfort, eds.), vol. 322 of Lecture Notes in Business Information Processing (LNBIP), pp. 202-220, Springer, 2018.

[17] F. E. Sandnes and T.-M. Grønli, "Thirty years of NIK: A bibliometric study of paper impact and changes in publication patterns," Norsk Informatikkonferanse, 2018.

[18] "AIS: elibrary)." https://aisel.aisnet.org/, 2019.

[19] "The advantage of short paper titles royal society open science." https:// royalsocietypublishing.org/doi/full/ 10.1098/rsos.150266, 2015. (Accessed on 06/14/2019).

[20] E. Orduna-Malea, A. Martín-Martín, M. Thelwall, and E. D. López-Cózar, "Do researchgate scores create ghost academic reputations?," Scientometrics, vol. 112, no. 1, pp. 443-460, 2017.

[21] "Scimago journal \& country rank." https: //www.scimagojr.com/, 2019. (Accessed on 06/14/2019)

[22] J. E. Hirsch, "An index to quantify an individual's scientific research output," Proceedings of the National academy of Sciences, vol. 102, no. 46, pp. 16569-16572, 2005.

[23] E. Marcotte, "Responsive Web Design." http: //alistapart.com/article/responsiveweb-design, 2013.

[24] Z. Q. Zhou, T. H. Tse, and M. Witheridge, "Metamorphic robustness testing: Exposing hidden defects in citation statistics and journal impact factors," IEEE Transactions on Software Engineering, pp. 1-1, 2019.

[25] T. Bui, ed., 50th Hawaii International Conference on System Sciences, HICSS 2017, Hilton Waikoloa Village, Hawaii, USA, January 4-7, 2017, ScholarSpace / AIS Electronic Library (AISeL), 2017. 